\title{
EDITORIAL
}

\section{La necesaria asunción de la prevención de riesgos laborales desde una perspectiva de género}

\author{
Eva M. BLÁZquEz Agudo \\ Fundadora de FEMERIS \\ eagudo@der-pr.uc3m.es
}

doi: https://doi.org/10.20318/femeris.2020.5151

La prevención de riesgos en la empresa no puede ser aplicada de la misma forma a toda la plantilla. Las medidas de seguridad y salud laboral tienen que ser adaptadas a las características no solo de los puestos, sino también de las personas trabajadora. En este sentido, la Ley de Prevención de Riesgos en su artículo 25 señala que la empresa debe garantizar la protección de los trabajadores que, por sus características personales o estado biológico conocido, sean especialmente sensibles a los riegos derivados del trabajo. Y, en concreto, se deben tener en cuenta en las evaluaciones los factores que puedan incidir en la función de procreación de las personas trabajadoras con el fin de adoptar las medidas preventivas precisas. Dentro de estas características personales o biológicas, como a continuación se expondrá, las trabajadoras tienen diferencias claras con los trabajadores, tanto desde el punto de vista del rol de la reproducción como del cuidado que deben ser tenidas en cuenta en la programación de la prevención a los efectos de evitar una situación de desventaja para las primeras en el mercado laboral. No significa esto que haya que entender que todas las mujeres deban ser tratadas como un colectivo especialmente sensible, pero sí que sus características deben ser tenidas en cuenta en la evaluación de riesgos y en las medidas establecidas a los efectos. Lo que es preciso es la inclusión de la perspectiva de género en la prevención de riesgos.

La Estrategia Europea en materia de Salud y Seguridad en el Trabajo 2014-2020 incluye medidas de salud y seguridad en el trabajo que pueden contribuir a luchar contra la discriminación y a promover la igualdad de oportunidades en las políticas de la Unión Europea; y la Directiva 2006/54/CE prohíbe el trato desfavorable de las mujeres en el lugar de trabajo por motivos de embarazo o maternidad. En el ámbito nacional, en la Constitución Española, cuando el párrafo segundo del artículo 40 reconoce la obligación 
de los poderes públicos de velar por la seguridad e higiene en el trabajo y el 43 reconoce a todos el derecho a la protección de la salud no hace mención expresa a ninguna diferencia de tratamiento por razón de género, no obstante, se puede inferir de su relación con los preceptos 9 y 14, que prohíben la desigualdad por razón de sexo. Y no se puede alcanzar la igualdad en el mercado laboral sin neutralizar todos los elementos que llevan a situar a las trabajadoras en plena igualdad, lo cual no puede alcanzarse, como se verá a continuación, sin la aplicación de la perspectiva de género en la prevención de riesgos laborales.

En este contexto, la Ley de 31/1995, de 8 de noviembre, de Prevención de Riesgos, trata especialmente la situación de las embarazadas y las lactantes en el centro de trabajo. La base de la protección de las trabajadoras se encuentra en la existencia de riesgos específicos para el embarazo, de modo que se impone la obligación de evaluar previamente los puestos de trabajo en una empresa con identificación específicamente de los riesgos, reales o potenciales, que supongan, o puedan suponer, un riesgo para la salud e integridad física de la embarazada o del feto. En el supuesto de que una trabajadora embarazada esté desarrollando este puesto previamente evaluado, habrá que adaptar las condiciones o del tiempo de trabajo o, en su caso, cambiar a esa trabajadora de puesto o función. Si ninguna de estas dos opciones es posible, entonces se suspenderá la relación laboral. Igual iter se sigue en el caso de que se detecten factores de riesgo derivados de las condiciones de trabajo de la madre, que se pueden transmitir al hijo a través de la leche materna.

Hay que poner de manifiesto que en el caso del embarazo lo que se valora son los riesgos de un puesto de trabajo concreto para todas las embarazadas y no si una mujer concreta debido a su propia situación física durante el estado de embarazo no puede desarrollar un trabajo concreto. En este supuesto, si la mujer no puede desarrollar su actividad por encontrarse en una situación física que no se lo permite, con independencia de la evaluación de su puesto, entonces solamente tendrá la posibilidad de solicitar una suspensión del contrato de trabajo por razón de incapacidad temporal, lo que le llevará a obtener una protección social inferior de la que se recibe en el caso de que finalmente no quede otra opción y se suspenda la actividad por riesgo durante el embarazo. En este último caso es la propia actividad la que es incompatible con dicha circunstancia, es en la que concurren riesgos propios para las embarazadas, aunque también se extiende a otros generales que pueden agravarse por dicho estado de embarazo.

Fuera de estas dos situaciones donde se tutela como colectivo sensible a las mujeres, la norma preventiva recoge, en el párrafo quinto del artículo 4 de la Ley de Prevención de Riesgos Laborales, la obligación de las Administraciones Públicas de promover la efectividad del principio de igualdad entre mujeres y hombres, considerando las variables relacionadas con el sexo tanto en los sistemas de recogida y tratamiento de datos como en el estudio e investigaciones generales en materia de prevención de riesgos laborales. Estos deberes tienen como objetivo de detectar y prevenir posibles situaciones en las que los daños derivados del trabajo puedan aparecer vinculados con el sexo de las personas trabajadoras. Así, se impone la aplicación de esta perspectiva en las políticas públicas en materia de prevención desde dos puntos de vista desde las diferencias biológicas, pero también socioeconómicas. Antes, las actividades preventivas se focalizaban en proteger 
a los trabajadores de los riesgos físicos o biológicos. Pero, sin embargo, no se recoge la misma obligación para las empresas.

En cierto modo, se trata de un mandato de integrar la perspectiva de género en la prevención para la Administración, pero no para las empresas. Sin embargo, como ya se adelantó, hay ciertas características de las trabajadoras que las diferencian con los trabajadores, las cuales deberían tenerse en cuenta en las evaluaciones de riesgos con el objeto de evitar ciertos elementos de desigualdad como puede ser el hecho de que las mujeres tengan una mayor tasa de absentismo por enfermedad común que los hombres, lo que puede ser un elemento negativo en su contratación. De hecho, las mujeres sufren más bajas por enfermedad común y menos accidentes de trabajo. Lo primero por las cuestiones que a continuación se van a analizar, la segunda seguramente debido a que son los sectores más masculinizados los que sufren una mayor siniestralidad. En todo caso, las enfermedades comunes son las que provocan un mayor número de procesos de baja.

Desde el punto de vista de las enfermedades comunes, hay varias situaciones que afectan a las mujeres que deben valorarse en la evaluación de riesgos y en las posteriores medidas aplicables. Es fundamental, en todas ellas, la valoración desde la perspectiva de género a los efectos de que las acciones preventivas sean adecuadas a las condiciones de las trabajadoras y que reduzcan el tipo de enfermedades por las que quedan más afectadas.

Así, en primer lugar, como ya se ha mencionado, fuera de los supuestos de riesgo durante el embarazo, el estado de gestación se trata como una enfermedad y, en su caso, da lugar a situaciones de baja por incapacidad temporal. Pero, además, las mujeres sufren las enfermedades con distinta incidencia que los hombres. Primeramente, quedan afectadas por las patologías ginecológicas, lo cual marca una diferencia en las medidas preventivas a aplicar por razones obvias. Pero, además, sus características biológicas llevan a que tengan un mayor número de procesos provocados por enfermedades concretas como los dolores musculoesqueléticos, que es la primera causa de baja por incapacidad temporal y que afectan más a las trabajadoras.

Por otra parte, las mujeres desarrollan actividades en determinados sectores concretos, consideradas especialmente feminizados, que conllevan ciertas enfermedades. Así, por ejemplo, las camareras de piso quedan afectan por enfermedades del sistema musculoesqueléticos; o las trabajadoras de los sectores de limpieza, peluquería y cosmética por las producidas por los riesgos a la exposición de productos químicos, tales como cierto tipo de cánceres u otras que influyen en el sistema hormonal. Por otra parte, las mujeres, en general, desarrollan actividades más precarias, de forma que están expuestas a riesgos laborales que no sufren los mandos intermedios.

A todas estas cuestiones, hay que añadir que la segunda causa de enfermedad de las trabajadoras son las patologías psiquiátricas, que engarzan con los riesgos psicosociales. Muchas de las situaciones que sufren las trabajadoras en el mercado laboral influyen en el mayor número de bajas por este motivo. Así, por ejemplo, el mayor riesgo que tienen de sufrir acoso sexual y violencia en el ámbito laboral, dado que trabajan en los sectores con mayor incidencia en este ámbito; las peores condiciones laborales y la doble jornada (en su puesto de trabajo y en su hogar en labores de cuidado). 
Si bien es verdad que las enfermedades provocadas por situaciones de acoso sexual y por razón de sexo tendrían que considerarse como derivadas del trabajo y, por tanto, ser calificadas como de origen profesional, sin embargo, en la mayoría de los casos, es difícil probar la relación entre la enfermedad y la actividad laboral y, por tanto, son consideradas como enfermedades comunes, lo que lleva a la consecución de bajas con una menor protección social. Todas estas enfermedades derivan de los riesgos psicosociales, que es el tema que a continuación se analiza.

Así, fuera del ámbito de la prevención de enfermedades y accidentes de trabajo, un ámbito fundamental es el de los factores y riesgos psicosociales, cuyo principal problema es su dificultad de valoración en las evaluaciones de riesgos. Se trata de daños derivados del trabajo, más allá de las enfermedades o los accidentes, que derivan de las condiciones psicosociales laborales disfuncionales, tales como el estrés, la violencia en el trabajo, el acoso psicológico, el acoso sexual o el burnout, entre otros, que, en general, afectan especialmente a las trabajadoras. La Agencia Europea de Seguridad y Salud en el Trabajo en 2007 reconoce 42 riesgos psicosociales dentro de cuatro grandes grupos: la inseguridad laboral, el conflicto trabajo-familia, el trabajo emocional y la intensificación de las demandas laborales. A las dos primeras causas se va a hacer mención en esta editorial.

La primera de ellas, la inseguridad laboral, está relacionada con la violencia laboral que es definida por la OIT como toda acción, incidente o comportamiento que se aparta de lo razonable en la cual una persona es asaltada, amenazada, humillada o lesionada como consecuencia directa de su trabajo. Como se ha señalado, las mujeres desarrollan su actividad laboral en los sectores que sufren más violencia laboral, tales como enseñanza, trabajo social, sanidad o el comercio minorista y, además, son las que están más expuestas en estas actividades, dado que no ocupan los puestos de trabajo de mando.

Pero, en este ámbito, también hay que tener en cuenta las situaciones menos explicitas de violencia como son el acoso psicológico en el trabajo o el sexual. La Ley Orgánica 3/2007, de 22 de marzo, para la Igualdad Efectiva de Mujeres y Hombres, introduce la definición de acoso sexual y acoso por razón de sexo. Se entiende por la primera cualquier comportamiento, verbal o físico, de naturaleza sexual que tenga el propósito o produzca el efecto de atentar contra la dignidad de una persona, en particular cuando se crea un entorno intimidatorio, degradante u ofensivo; mientras que se define acoso por razón de sexo como cualquier comportamiento realizado en función del sexo de una persona, con el propósito o el efecto de atentar contra su dignidad y de crear un entorno intimidatorio, degradante u ofensivo. Ambos son discriminatorios por razón de sexo y existe una obligación de las empresas de prevenir estas situaciones y remediarlas a través de procedimientos específicos para prevención y para dar cauce a denuncias o reclamaciones. Además, tienen la obligación de negociar con los representantes de los trabajadores la elaboración y difusión de códigos de buenas prácticas, así como la puesta en marcha de campañas informativas o acciones de formación. Estos a su vez tienen el deber de contribuir a su prevención mediante la sensibilización, así como de informar a la empresa sobre las conductas o comportamientos de que tuvieran conocimiento y que pudieran propiciar. 
Desde aquí son precisas, además de las medidas de prevención, otras de remedio de las situaciones. Respecto al acoso sexual, es preciso la negociación de protocolos en la empresa que permitan identificar las situaciones de esta naturaleza y que resuelvan satisfactoriamente la situación. Si las medidas de prevención no han evitado el acoso sexual, al menos las de remedio deberían imponer sanciones al acosador y asegurar a la víctima la continuación de su actividad laboral en condiciones óptimas que eviten posibles enfermedades psiquiátricas que puedan derivar de la irresolución del conflicto. Esto es, los protocolos de acoso son, en cierto modo, también medidas preventivas de enfermedades que afectan en mayor medida a las mujeres debido a la incidencia de estas situaciones.

Respecto al acoso por razón de sexo, la evitación de situaciones de desigualdad en la empresa, tales como, por ejemplo, menores posibilidades de promoción también actúan como medidas preventivas de patologías psiquiátricas. Obviamente el mejor cauce para este tipo de acciones son los Planes de Igualdad más que las acciones preventivas de riesgos laborales, pero, en su caso, deberían coordinarse.

Entrando en la segunda causa, el conflicto trabajo-familia, este también provoca efectos perniciosos en la salud y bienestar de las trabajadoras. Dichos conflictos influyen desde dos puntos de vista, cuando las actividades relacionadas con el trabajo dificultan el desempeño de las responsabilidades familiares, por ejemplo, cuando la persona trabajadora se lleva trabajo a casa, impidiendo el descanso o el tiempo en familia; o cuando las responsabilidades familiares son las que impiden el desarrollo correcto de la actividad laboral, por ejemplo, cuando se tiene que cuidar a un familiar enfermo. Estas situaciones deben ser atendidas por medidas que traten de resolver estas cuestiones, más allá de la prevención de riesgos, que pasan por acciones de corresponsabilidad que permitan la flexibilidad de la jornada y su adaptación a las necesidades familiares, especialmente a través de los Planes de Igualdad en la empresa. En todo caso, estos conflictos pueden provocar un menor nivel de satisfacción laboral, que de nuevo influye en la salud de las trabajadoras. Igual que se señaló en relación con el acoso por razón de sexo, estas cuestiones deben ser atajadas por los Planes de Igualdad, que, en todo caso, debe contener medidas conectadas con las acciones de prevención de riesgos laborales.

En resumen, es preciso que la empresa emprenda la evaluación de riesgos laborales y la determinación de medidas preventivas teniendo en cuenta la perspectiva de género a los efectos de adaptar estas acciones a las características físicas y sociales de las trabajadoras que no son las mismas que las de los trabajadores. 